\title{
Nanostenciling for fabrication and interconnection of nanopatterns and microelectrodes
}

\author{
Haiming Guo, David Martrou, Tomaso Zambelli, Jérôme Polesel-Maris, Agnès Piednoir, \\ Erik Dujardin, and Sébastien Gauthier ${ }^{\mathrm{a})}$ \\ Centre d'Elaboration de Matériaux et d'Etudes Structurales-CNRS BP 94347, \\ 31055 Toulouse Cedex 4, France
}

\author{
Marc A. F. van den Boogaart, Lianne M. Doeswijk, and Jürgen Brugger \\ Ecole Polytechnique Fédérale de Lausanne (EPFL), Laboratoire de Microsystèmes, \\ CH-1015 Lausanne, Switzerland
}

(Received 27 November 2006; accepted 25 January 2007; published online 28 February 2007)

\begin{abstract}
Stencil lithography is used for patterning and connecting nanostructures with metallic microelectrodes in ultrahigh vacuum. Microelectrodes are fabricated by static stencil deposition through a thin silicon nitride membrane. Arbitrary nanoscale patterns are then deposited at a predefined position relative to the microelectrodes, using as a movable stencil mask an atomic force microscopy (AFM) cantilever in which apertures have been drilled by focused ion beam. Large scale AFM imaging, combined with the use of a high precision positioning table, allows inspecting the microelectrodes and positioning the nanoscale pattern with accuracy better than $100 \mathrm{~nm}$. (C) 2007 American Institute of Physics. [DOI: 10.1063/1.2710473]
\end{abstract}

In spite of a vast number of attempts, the electrical addressing of nanoscale or single molecular systems remains a major technological challenge in nanoelectronics and molecular electronics. On the one hand, scanning probe microscopy ${ }^{1}$ (SPM) and, to a lesser extent, break junctions ${ }^{2}$ currently offer the best stability and accuracy to investigate local electrical properties of a limited number of nanometerscale systems. However, even recent strategies to implement four-probe addressing tools within SPM setups ${ }^{3}$ or gates in break junctions ${ }^{4}$ do not provide for a viable method to integrate single nanodevices into complex integrated circuits. On the other hand, parallel techniques such as crossbar or lithographically prepared electrode junctions provide direct transport measurements on assemblies of molecules. ${ }^{5,6}$ Nevertheless, these approaches are still hampered by the lack of control of the metal/molecule interface, in particular, owing to the presence of residual polymeric resists. In this context, there is a clear need to develop original parallel and resistless approaches for defining planar electrical connections from the macroscopic level of integrated circuits down to the level of nanoscale single objects. This span of length scales generates two antagonistic challenges. First, addressing truly nanometer-scale systems requires the fabrication of electrodes down to a few nanometers in both width and spacing without altering or contaminating the targeted object. Secondly, a precise and compact electrode design is necessary to connect these nanojunctions to microelectrodes and external measuring circuits.

Recently, the second of these challenges was successfully overcome by the stencil lithography, in which a variety of materials can be evaporated directly through a membranesupported aperture onto a substrate in an ultraclean environment. Stenciling provides a direct, simple, low-cost, and resistless fabrication tool for patterning complex structures with well-defined geometries from the micrometer range down to $10 \mathrm{~nm}$ without detrimental effects on the

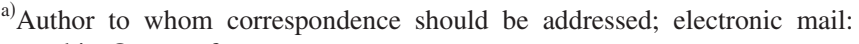
gauthier@cemes.fr
}

substrate. ${ }^{7-11}$ Although the aperture shape can be chosen arbitrarily provided it is compatible with the supported membrane geometry, this approach is essentially static in the sense that the deposited pattern is the replica of the predefined aperture. A recent development is the dynamic stencil lithography, ${ }^{12}$ in which the stencil mask is embedded in an atomic force microscopy (AFM) cantilever. In this case, an arbitrarily designed deposit can be obtained by controlling the motion of a simple cantilever-borne mask, such as a small hole or a narrow slit drilled by focused ion beam (FIB), with respect to the sample. Another benefit of stenciling with a FIB-drilled AFM tip is to directly image the surface where the nanopattern will be evaporated, which is crucial to achieve nanometer-scale alignment between two stencil levels.

In this letter, we present a technique combining two stencil lithography steps, the first one with a static stencil mask and the second one with a movable AFM cantilever. Microelectrodes are fabricated on insulating substrates by static stencil deposition through a thin membrane. After in situ characterization by large scale AFM imaging, the desired nanostructures are deposited between the microelectrodes using the apertures drilled in the AFM cantilever.

Figure 1 gives a schematic view of our setup developed by modifying a variable temperature AFM/scanning tunneling microscope (STM) Omicron Nanotechnology head. Details will be described in Ref. 13. Briefly, the sample is placed on an $X-Y$ nanopositioning table (Piezosystem Jena $\mathrm{GmbH}$, Germany), equipped with capacitive sensors actuated by piezoceramics in a closed feedback loop configuration, providing a repeatability of $20 \mathrm{~nm}$ on a $100 \times 100 \mu \mathrm{m}^{2}$ area. This table enables the accurate positioning of the cantilever with respect to the microelectrodes for dynamic stencil deposition. It also allows large scale AFM imaging of the surface, which is necessary to go beyond the small scanning area of the Omicron head $\left(5 \times 5 \mu \mathrm{m}^{2}\right)$. The deposit is obtained from an effusion cell by collimating the evaporation beam on the FIB-drilled AFM cantilever by a series of three diaphragms with decreasing diameters. The smallest diaphragm $(0.5 \mathrm{~mm}$ 


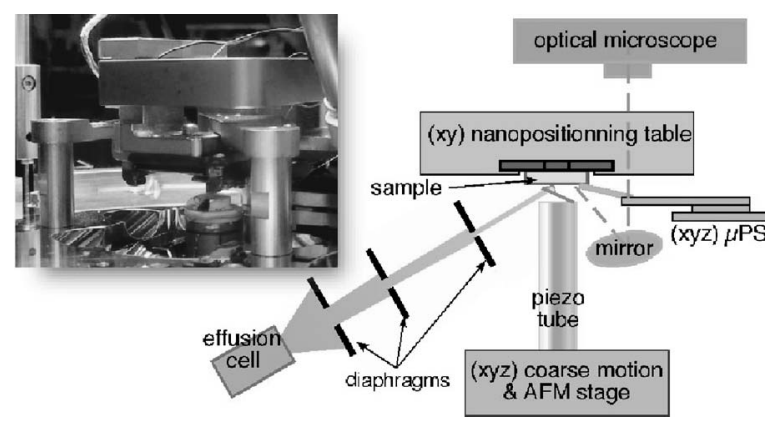

FIG. 1. Schematic diagram of the experimental setup. The substrate is mounted on an $(X Y)$ nanopositioning stage, and the collimated metal vapor beam is guided from the evaporator to the substrate through the apertures in the AFM cantilever. An $X-Y-Z$ micropositioning stage (XYZ- $\mu$ PS) allows the positioning of the microcantilever array, which can be viewed by an optical microscope. Inset: Photography of the modified VT AFM/STM Omicron head.

diameter) is mounted on a two axis piezotable so that the metallic beam spot can be positioned at will. In addition, an array of ten metallic cantilevers can be brought in contact with the patterned microelectrodes thanks to a $X-Y-Z$ micropositioning stage in order to electrically connect the nanodevices to external measuring instruments. ${ }^{14}$

The static stencil masks used for patterning the microelectrodes were fabricated in silicon nitride membranes (size: $1 \times 1 \mathrm{~mm}^{2}$, thickness: $500 \mathrm{~nm}$ ) by advanced microelectromechanical system processing, as described elsewhere. ${ }^{8}$ The UHV environment required the addition of $1 \mu \mathrm{m}$ thick $\mathrm{Al}$ spacers on the periphery of the membrane. These spacers reproducibly maintain a constant gap between the mask and the substrate while avoiding the sticking of the mask to the sample surface during evaporation. Each membrane was patterned with a $9 \times 9$ array of microelectrodes patterns, as shown in Fig. 2(a). Importantly, the microelectrodes were designed so that the specific microelectrode pattern under consideration could be protected during nanostencil deposition by the shadow of the cantilever, as shown in Fig. 2(b). Depending on the selected microelectrode pattern in the $9 \times 9$ array, the other patterns can be protected or not.

The nanostencil apertures were drilled in AFM tip walls by FIB. ${ }^{15}$ In order to minimize charging effects under the ion beam contact $\mathrm{AFM} \mathrm{Si}_{3} \mathrm{~N}_{4}$ cantilevers with top and bottom side gold/chromium coatings were selected. ${ }^{16}$ Apertures were performed in the rear face of the square pyramidal tip [Fig. 3(a)]. A two-step protocol was developed which consisted in (i) thinning the approximately $800 \mathrm{~nm}$ thick tip wall
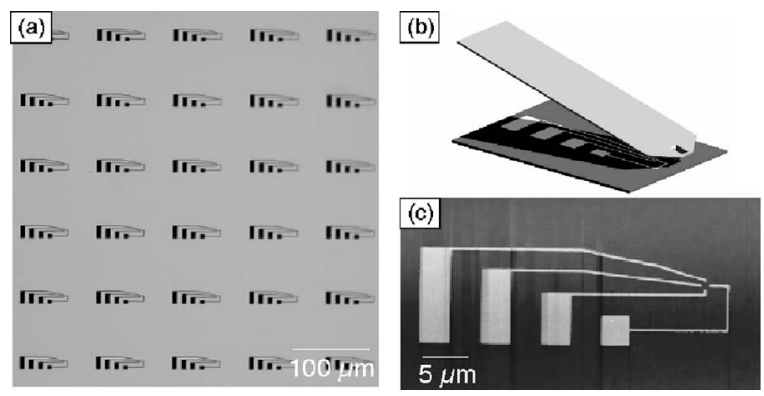

FIG. 2. (a) Optical microscopy images of silicon nitride membranes with microelectrodes patterns. (b) Three-dimensional view of the microelectrodes protected by the shadow of the AFM cantilever. (c) Contact AFM image of $\mathrm{Au}$ microelectrodes deposited on a $\mathrm{SiO}_{2}$ substrate through a static stencil

AFM mode. After having deposited a metal film, large scale
Downloaded 01 Mar 2007 to 128.178.153.159. Redistribution subject to AIP license or copyright, see http://apl.aip.org/apl/copyright.jsp
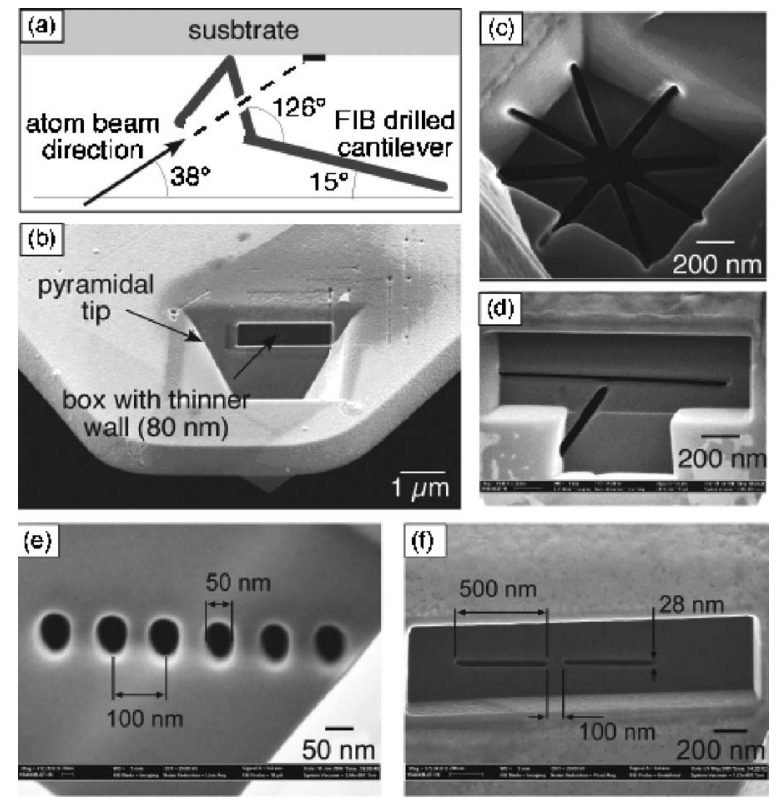

FIG. 3. (a) Schematic view of the geometrical configuration of the AFM nanostencil technique. (b) Scanning electron microscopy image showing a recessed box in the rear face of AFM tip, which was first thinned down to $80 \mathrm{~nm}$ by FIB. (c) The cross apertures are drilled in the AFM tip wall. (d) A two lines pattern with $52^{\circ}$ angle necessary to correct the geometrical distortions due to the evaporation geometry. (e) A line of holes. (f) An interrupted slit.

down to approximately $80 \mathrm{~nm}$ at a nominal ion current of 10 pA [Fig. 3(b)] and (ii) drilling the designed apertures with sub-100 nm lateral dimensions at a nominal ion current of 1 pA [Figs. 3(c) and 3(d)]. This protocol ensures that aperture features of less than $30 \mathrm{~nm}$ lateral size can be reproducibly and routinely achieved. To limit the extension of the penumbra of the evaporated pattern, the aperture in the tip wall should be positioned as close as possible to the surface of the sample. The minimal distance which can be achieved, due to geometrical constraints, is of the order of $2 \mu \mathrm{m}$ between the aperture and the tip apex. In these conditions, a simple calculation gives a penumbra of $20 \mathrm{~nm}$. Obviously, if the deposition is made in the noncontact AFM mode, a broadening of the order of the oscillation amplitude, that is, $10 \mathrm{~nm}$, has to be added to this penumbra.

The stenciling procedure starts by patterning the microelectrodes with the static mask. For this, the stencil mask is brought close to the surface by a dedicated wedged mechanism until the $1 \mu \mathrm{m} \mathrm{Al}$ spacers touch the surface. The metal crucible is located at $80 \mathrm{~mm}$ in a direction perpendicular to the substrate. Figure 2(c) shows an in situ AFM image of $13 \mathrm{~nm}$ thick $\mathrm{Au}$ microelectrodes deposited on a $\mathrm{SiO}_{2}$ substrate at room temperature. The pattern and the crosssectional profile of the electrodes agree well with the deposition geometries of the stencil system. The broadening is negligible and leaves an intact central area of $1.5 \times 1.5 \mu \mathrm{m}^{2}$.

The nanopatterning of the intermicroelectrode area by the nanostencil technique relies on the precise determination of the actual location of the deposited nanopattern with respect to the nanostencil AFM tip. Consequently, a preliminary calibration is needed for each apertured AFM cantilever. In a typical calibration experiment, the cantilever is approached to a bare substrate in either noncontact or contact AlP license or copyright, see http://apl.aip.org/apl/copyright.jsp 

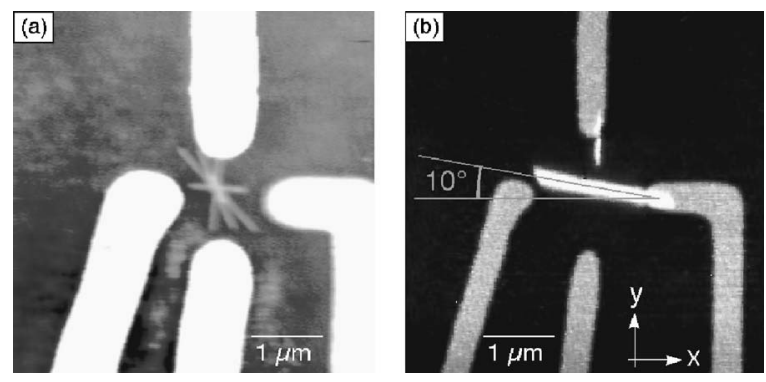

FIG. 4. Noncontact AFM images of nanopatterns deposited between the microelectrodes $\left(5 \times 5 \mu \mathrm{m}^{2}\right)$. Imaging conditions: (a) Amplitude $=8.6 \mathrm{~nm}$, $\Delta f=-20 \mathrm{~Hz}$, scanning speed $=1 \mu \mathrm{m} / \mathrm{s}$; (b) amplitude $=13 \mathrm{~nm}, \Delta f=-40 \mathrm{~Hz}$, scanning speed $=2.5 \mu \mathrm{m} / \mathrm{s}$.

AFM images are acquired by scanning the $X-Y$ table and the relative position of the deposited pattern and the AFM tip can be precisely measured. Subsequently, the bare substrate is exchanged with the substrate with predeposited microelectrodes. Knowing the location of one set of microelectrodes and the relative position of the deposit with respect to the AFM tip apex, the nanopositioning table is moved so as to deposit the nanopatterns precisely between the microelectrodes.

Figure 4(a) shows a noncontact AFM image of a $\mathrm{Cu}$ cross nanopattern between gold microelectrodes on a thermal silicon oxide substrate obtained from the nanostencil mask from Fig. 3(c). The comparison of the mask deposit patterns gives a complete characterization of the geometrical distortions of our system, resulting from the AFM cantilever tilt angle $\left(15^{\circ}\right)$, the angle of the pyramidal tip $\left(126^{\circ}\right)$, and the atom beam direction $\left(38^{\circ}\right)$, as shown in Fig. 3(a). To compensate for this projection, a transformation of the desired pattern is applied to the drilled aperture pattern. For example, an orthogonal cross pattern of $5 \mathrm{~nm}$ thick $\mathrm{Cu}$ lines centered between Pd microelectrodes [Fig. 4(b)] is obtained by evaporation through the modified aperture shown in Fig. 3(d). These two examples show the difficulties met for precise aligning: the cross is misaligned by $100 \mathrm{~nm}$ in both $x$ and $y$ directions, while the second pattern is correctly aligned in $y$ direction but not in the $x$ direction, and a residual angle of $10^{\circ}$ is observed [Fig. 4(b)]. The positioning error in $x$ and $y$ is directly related to the uncertainty in measuring the tip to nanopattern distance, most probably due to tip alteration during scanning. This effect is strongly decreased when performing imaging in noncontact mode. The angular discrepancy results from angular errors in tip clamping on the two tip holders used for FIB milling and AFM imaging. A more careful design of both tip holders can minimize these errors in order to reach the optimal accuracy of $20 \mathrm{~nm}$ provided by the $X-Y$ table. In terms of feature resolution, Fig. 4(b) shows that the narrowest $\mathrm{Cu}$ wires are $80 \mathrm{~nm}$ full width at half maximum, for pattern heights of typically $5-7 \mathrm{~nm}$ in the direction of smaller geometrical broadening (direction $x$ ).

Figure 4 shows patterns obtained using the cantileverborne stencil in a static configuration, i.e., without moving the substrate during deposition. Proceeding further, our twostage nanostenciling method has the potential to draw nanopatterns by moving the stage while evaporating through a single hole stencil. This includes features not achievable by membrane stencils such as circles. ${ }^{11}$ A drawback of this dynamic method is that the AFM cantilever is exposed to the evaporation beam for a much longer time when drawing complex patterns than when depositing the same pattern through a static complex stencil, leading to clogging of the aperture. A clogging test during silver evaporation through the circular apertures shows that the clogging thickness of silver is approximately equal to the aperture diameter for sub-100 nm stencil deposition, confirming previous findings. ${ }^{17}$ Anticlogging surface treatment reported for static stenciling ${ }^{14}$ is currently being implemented on cantileverborne stencils. A practical compromise consists in designing simple aperture patterns, such as slits or hole arrays [Figs. $3(\mathrm{e})$ and 3(f)] that can be translated to create more complex pattern while keeping the exposure time to a minimum. ${ }^{4}$

In summary, we have developed a two-step stencil lithography process which allows positioning nanoscale structures relative to microelectrodes in a direct and clean way under UHV environment. The positioning accuracy is presently of the order of $100 \mathrm{~nm}$, but may be eventually improved to $20 \mathrm{~nm}$. Along with the microcantilever array, this setup covers the full range from nanometer- up to millimeterscale connections to contact the nanoelectrodes to external measuring instruments. This technique thus provides a promising way for electrical measurements of nanoscale and molecular devices in UHV environment.

The authors would like to thank L. Guiraud and M. A. Venegas for fruitful discussions and technical help. Partial support by the French Ministry of Research (Décision d'Aide No. $00 \mathrm{~V}$ 0068), the European Commission within the project CHIC (Contract No. IST-2001-33578), and the European Commission and the Swiss Federal Office for Education and Science (OFES) within the project $\mathrm{NaPa}$ (Contract No. NMP4-CT-2003-500120) is gratefully acknowledged. The content of this work is the sole responsibility of the authors.

${ }^{1}$ C. Joachim, J. K. Gimzewski, R. R. Schlittler, and C. Chavy, Phys. Rev. Lett. 74, 2102 (1995).

${ }^{2}$ J. Reichert, R. Ochs, D. Beckmann, H. Weber, M. Mayor, and H. V. Löhneysen, Phys. Rev. Lett. 88, 176804 (2002).

${ }^{3}$ X. Lin, X. He, T. Yang, W. Guo, D. Shi, H. Gao, D. Ma, S. Lee, F. Liu, and X. Xie, Appl. Phys. Lett. 89, 043103 (2006).

${ }^{4}$ A. R. Champagne, A. N. Pasupathy, and D. C. Ralph, Nano Lett. 5, 305 (2005).

${ }^{5}$ D. R. Stewart, D. A. A. Ohlberg, P. A. Beck, Y. Chen, R. S. Williams, J. O. Jeppesen, K. A. Nielsen, and J. F. Stoddart, Nano Lett. 4, 133 (2004).

${ }^{6}$ M. S. M. Saifullah, T. Ondarcuhu, D. K. Koltsov, C. Joachim, and M. E. Welland, Nanotechnology 13, 659 (2002).

${ }^{7}$ M. M. Deshmukh, D. C. Ralph, M. Thomas, and J. Silcox, Appl. Phys. Lett. 75, 1631 (1999).

${ }^{8}$ M. A. F. van den Boogaart, G. M. Kim, R. Pellens, J. P. van den Heuvel, and J. Brugger, J. Vac. Sci. Technol. B 22, 3174 (2004).

${ }^{9}$ S. Egger, A. Llie, T. Fu, J. Chongsathien, D. Kang, and M. E. Welland, Nano Lett. 5, 15 (2005).

${ }^{10}$ P. Zahl, M. Bammerlin, G. Meyer, and R. R. Schlittler, Rev. Sci. Instrum. 76, 023707 (2005).

${ }^{11}$ S. Egger, S. Higuchi, and T. Nakayama, J. Comb. Chem. 8, 275 (2006).

${ }^{12}$ R. Lüthi, R. R. Schittler, J. Brugger, P. Vettiger, M. E. Welland, and J. K. Gimzewski, Appl. Phys. Lett. 75, 1314 (1999).

${ }^{13}$ H. Guo, D. Martrou, M. J. Polesel-Maris, M. A. Venegas, T. Zambelli, E. Dujardin, L. Guiraud, and S. Gauthier, (unpublished).

${ }^{14}$ T. Ondarçuhu, L. Nicu, S. Cholet, C. Bergaud, S. Gerdes, and C. Joachim, Rev. Sci. Instrum. 71, 2087 (2000).

${ }^{15}$ Zeiss 1540 ExB Crossbeam FIB-FEGSEM, fitted with a Ga source and operated at $30 \mathrm{kV}$.

${ }^{16}$ OMCL-RC800PB from Olympus Corporation. These tips are hollow, with about $800 \mathrm{~nm}$ thick walls. Nominal resonance frequency $=66 \mathrm{kHz}$, nominal stiffness $=0.82 \mathrm{~N} / \mathrm{m}$.

${ }^{17}$ M. Kolbel, R. W. Tjerkstra, J. Brugger, C. J. M. van Rijn, W. Nijdam, J. Huskens, and D. N. Reinhoudt, Nano Lett. 2, 1339 (2002). 